\title{
Complete regression of metastatic malignant melanoma endoscopically after nivolumab administration
}

\author{
Dae Gon Ryu, Cheol Woong Choi, Su Jin Kim, Dae Hwan Kang, and Su Bum Park
}

Department of Internal Medicine, Medical Research Institute, Pusan National University School of Medicine and Research Institute for Convergence of Biomedical Science and Technology, Pusan National University Yangsan Hospital, Yangsan, Korea

Received: November 20, 2020 Revised : December 3, 2020 Accepted: December 4, 2020

\section{Correspondence to} Cheol Woong Choi, M.D.

Tel: +82-55-360-2360

Fax: +82-55-360-1536

E-mail: drluckyace@gmail.com https://orcid.org/0000-00018867-3039

Figure 1. (A) On positron emission tomography and computed tomography (PET-CT), brain metastasis and multiple liver, lung, bone, and pancreatic metastasis were observed. (B) Multiple liver, pancreatic, and lymph node metastasis were observed on abdominal CT. (C) Nodules of various sizes were observed in upper endoscopy. (D) A small nodule and pigmentations were observed at the recto-sigmoid junction on colonoscopy. (E) Round pleomorphic cells with large nuclei were observed (H\&E, ×400). (F) The cells showed immunohistochemical positivity for Melan A (×400).
A 64-year-old man presented with multiple metastatic malignant masses throughout the body. (Fig. $1 \mathrm{~A}$ and 1B). Endoscopic examination revealed umbilicated nodules of various sizes in the stomach (Fig. 1C) and a nodule with peripheral pigmentation in the rectum (Fig. 1D). Malignant melanoma was diagnosed in all endoscopic forceps biopsies (Fig. $1 \mathrm{E}$ and ${ }_{1} \mathrm{~F}$ ). He was diagnosed with malignant melanoma with multiple metastases and an unclear primary site. Nivolumab treatment (human programmed death receptor-1 [PD-1] blocking antibody) was started at $3 \mathrm{mg} / \mathrm{kg}$ every 2 weeks. Most metastases and endoscopic malignant nodules disappeared after 6 months of treatment (Fig. 2).

Malignant melanoma can metastasize to all parts of the body and the metastatic rate of the gastrointestinal tract is reportedly around 60\%. Initially, metastatic malignant melanoma did not respond to chemotherapy and was considered incurable. However, the advent of immunotherapy dramatically impacted the treatment response of malignant metastatic melanoma. We present comparative pictures showing complete endoscopic regression after PD-1 inhibitor treatment. The patient provided informed consent for publication of this case.

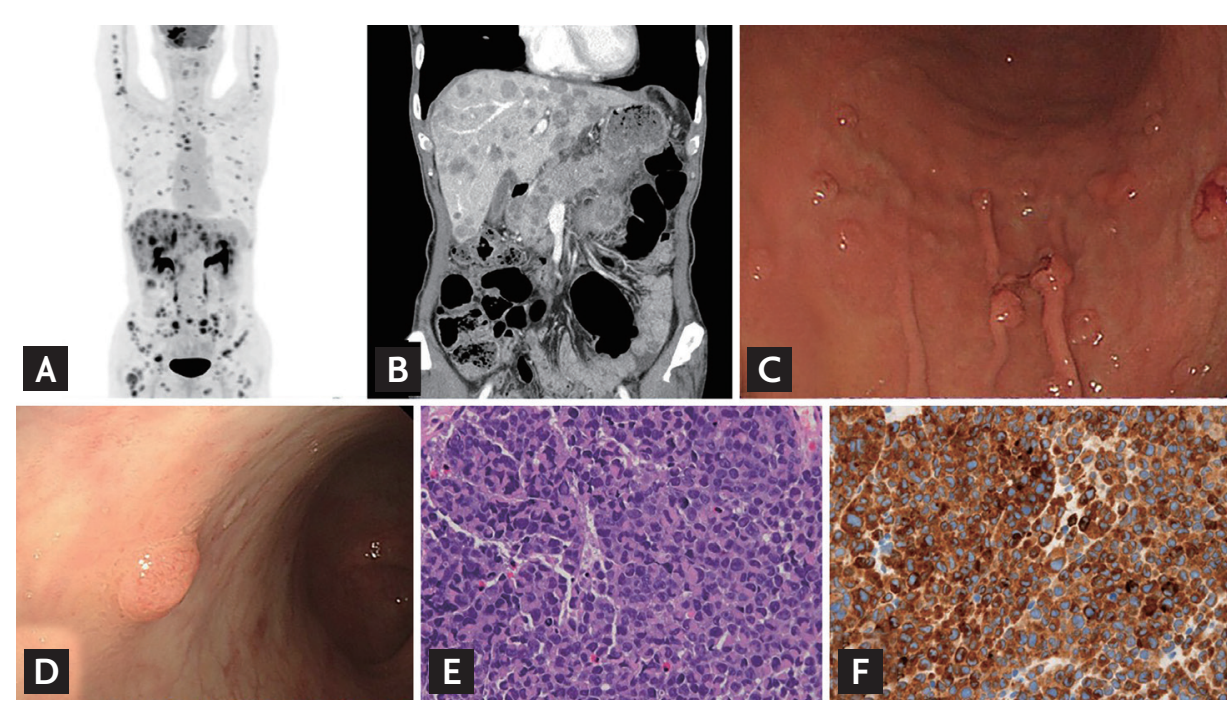



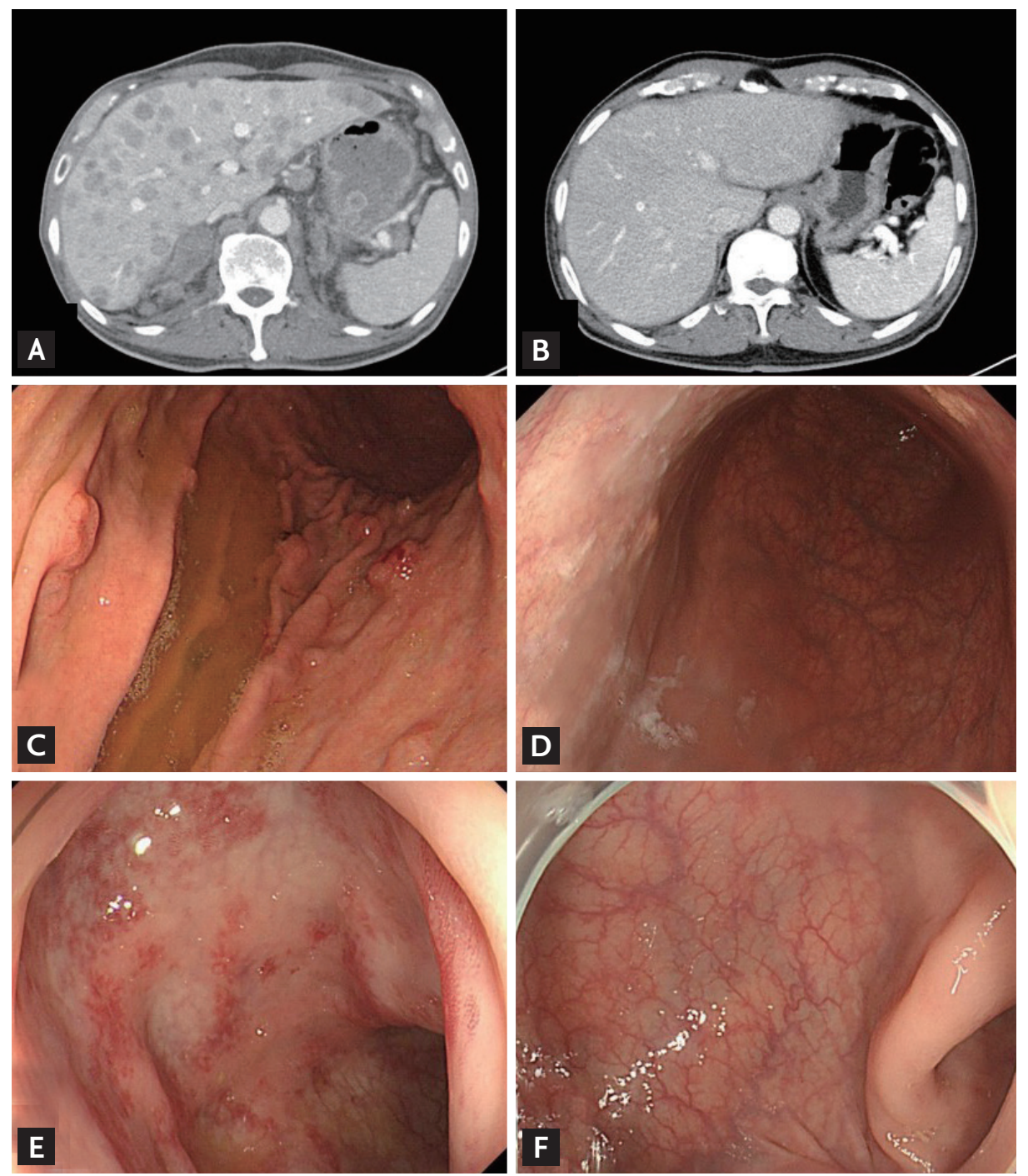

Figure 2. Comparative figures after 6 months of nivolumab administration. (A, B) The liver metastasis almost disappeared on abdominal computed tomography. (C, D) All gastric nodules disappeared. (E, F) Pigmentations at the recto-sigmoid junction were also not observed.

\section{Conflict of interest}

No potential conflict of interest relevant to this article was reported. 